\title{
Spondyloarthropathy and rheumatoid arthritis: two different diseases, a similar cytokine network
}

\author{
R A Moura ${ }^{1 \dagger}$, R Cascão $^{1+}{ }^{1+}$, Perpétuo ${ }^{1}$, H Canhão ${ }^{1,2}$, E Vieira de Sousa ${ }^{1,2}$, A F Mourão ${ }^{1,3}$, A M Rodrigues ${ }^{1,2}$, \\ J Polido-Pereira ${ }^{1,2}$, J A Pereira da Silva ${ }^{2}$, H S Rosário ${ }^{4}$, M M Souto-Carneiro ${ }^{5}$, L Graca ${ }^{6,7^{*}}$, J E Fonseca ${ }^{1,2^{*}}$ \\ From 5th European Workshop on Immune-Mediated Inflammatory Diseases \\ Sitges-Barcelona, Spain. 1-3 December 2010
}

\section{Introduction}

The reasons for the phenotypic differences between spondyloarthritis (SpA) and rheumatoid arthritis (RA) are still unclear. Slight divergences in the cytokine networks might contribute to this and may be the key to new treatment approaches.

\section{Aim}

The main goal of this work was to compare serum and synovial cytokines and chemokines in established SpA and RA patients.

\section{Patients and methods}

IL-1ß, IL-2, IL-4, IL-6, IL-8, IL-10, IL-12 (p70), IL-17A, IL-18, IL-21, IL-22, IL-23, IFN- $\gamma$, MCP-1, MIP-1 $\alpha$, OPG, sRANKL, TGF- $\beta$, TNF, APRIL and BAFF levels were measured in $\mathrm{SpA}(\mathrm{n}=8)$ serum and compared with RA $(\mathrm{n}=10)$ and controls $(\mathrm{n}=24)$. Synovial fluid $(\mathrm{SF})$ from SpA $(n=8)$, RA $(n=12)$ and osteoarthritis (OA) $(n=10)$ patients were also analyzed.

\section{Results}

No significant differences were found between SpA and RA, with the exception of higher levels of IL-21 in the SF of RA patients.

\section{Conclusions}

Our results suggest that although $\mathrm{SpA}$ and RA are chronic inflammatory diseases with different clinical manifestations, both share a similar cytokine profile, including Th17-related cytokines.

\begin{abstract}
Author details
${ }^{1}$ Rheumatology Research Unit, Instituto de Medicina Molecular, Faculdade de Medicina da Universidade de Lisboa, Lisbon, Portugal. ${ }^{2}$ Rheumatology Dept., Centro Hospitalar de Lisboa Norte, EPE, Hospital de Santa Maria, Lisbon, Portugal. ${ }^{3}$ Rheumatology Dept., Centro Hospitalar de Lisboa Ocidental, EPE, Hospital Egas Moniz, Lisbon, Portugal. ${ }^{4}$ Microvascular Biology and Inflammation Unit, Instituto de Medicina Molecular, Faculdade de Medicina da Universidade de Lisboa, Lisbon, Portugal. ${ }^{5}$ Center for Neurosciences and Cell Biology, Autoimmunity Group, Coimbra, Portugal. ${ }^{6}$ Cellular Immunology Unit, Instituto de Medicina Molecular, Faculdade de Medicina da Universidade de Lisboa, Lisbon, Portugal. ${ }^{7}$ Instituto Gulbenkian de Ciência, Oeiras, Portugal.
\end{abstract}

Published: 25 November 2010

\section{doi:10.1186/1479-5876-8-S1-P36}

Cite this article as: Moura et al:: Spondyloarthropathy and rheumatoid arthritis: two different diseases, a similar cytokine network. Journal of Translational Medicine 2010 8(Suppl 1):P36.

\footnotetext{
† Contributed equally

${ }^{1}$ Rheumatology Research Unit, Instituto de Medicina Molecular, Faculdade de Medicina da Universidade de Lisboa, Lisbon, Portugal

${ }^{6}$ Cellular Immunology Unit, Instituto de Medicina Molecular, Faculdade de

Medicina da Universidade de Lisboa, Lisbon, Portugal

Full list of author information is available at the end of the article
} 